\title{
Protée
}

\section{L’interaction métaphorique : une grandeur algébrique}

\section{Michele Prandi}

Volume 38, numéro 1, printemps 2010

Le Groupe $\mu$ entre rhétorique et sémiotique

URI : https://id.erudit.org/iderudit/039704ar

DOI : https://doi.org/10.7202/039704ar

Aller au sommaire du numéro

Éditeur(s)

Département des arts et lettres - Université du Québec à Chicoutimi

\section{ISSN}

0300-3523 (imprimé)

1708-2307 (numérique)

Découvrir la revue

Citer cet article

Prandi, M. (2010). L’interaction métaphorique : une grandeur algébrique. Protée, 38(1), 75-84. https://doi.org/10.7202/039704ar

\section{Résumé de l'article}

Il y a beaucoup de théories de la métaphore, et la raison est que la métaphore a une source unique, mais elle admet plusieurs issues différenciées, même opposées. À la différence d'une métonymie, qui relie des concepts saturés dans une relation cohérente, une métaphore naît du transfert d'un concept dans un domaine étranger. Le transfert provoque une interaction entre deux concepts qui se disputent le même objet : un sujet primaire et un sujet subsidiaire. Or, l'interaction est une grandeur algébrique, qui admet un solde négatif, un solde nul et un solde positif. Le solde négatif correspond à la catachrèse lexicale : le sujet subsidiaire se plie au profil conceptuel cohérent du sujet primaire. Le solde nul correspond à la substitution : le sujet primaire remplace le sujet subsidiaire, et toute interaction est bloquée. Le solde positif correspond à la projection : le sujet subsidiaire est projeté sur le sujet primaire ; de ce fait, il met en question son profil conceptuel et le redessine. La projection est une grandeur graduée, qui s'étend de l'activation de stéréotypes routiniers ou d'analogies évidentes jusqu'aux issues les plus surprenantes. À l'intérieur de ce continuum, le seuil critique est représenté par la condition de cohérence conceptuelle, qui sépare les concepts métaphoriques partagés, c'est-à-dire les "métaphores de la vie quotidienne », des métaphores vives et conflictuelles.
Ce document est protégé par la loi sur le droit d'auteur. L’utilisation des services d’Érudit (y compris la reproduction) est assujettie à sa politique d'utilisation que vous pouvez consulter en ligne.

https://apropos.erudit.org/fr/usagers/politique-dutilisation/ 


\section{L'INTERACTION MÉTAPHORIQUE: UNE GRANDEUR ALGÉBRIQUE}

MiCHELE PRANDI

Ce qui nous étonne quand nous pensons à la métaphore, c'est le grand nombre de théories différentes et souvent opposées qui ont été proposées le long des siècles. Dans l'histoire de la pensée, la métaphore a été définie à tour de rôle comme transfert de mot (Aristote, Poétique) et comme extension de sens d'un mot (Dumarsais, [1730] 1988), comme substitut (Fontanier, 1968; Genette, 1968; Groupe ，1970; Todorov, 1970) et comme interaction conceptuelle (Richards, 1936; Black, 1954 et 1967), comme système de concepts au service de la pensée cohérente (Blumenberg, 1949; Weinrich, 1958 et 1964; Lakoff et Johnson, 1981; Lakoff et Turner, 1989; Gibbs, 1994) et comme interprétation discursive d'un signifié incohérent (Weinrich, 1963 et 1967; Prandi, 1992). De toutes ces théories, aucune n'est complètement fausse, aucune n'est exhaustive. Toutes sont appuyées par des faits pertinents, aucune n'est en mesure de les inclure tous.

Ce que toutes ces théories différentes ont en commun, c'est de se concentrer sur l'une des issues admises par un processus métaphorique. Or, les issues d'un processus métaphorique sont nombreuses, différenciées et souvent même opposées, mais se développent toutes à partir d'une seule source, à savoir d'un transfert qui déclenche une interaction conceptuelle. Si nous isolons les issues, l'analyse de la métaphore est ou incohérente ou partielle, et une description adéquate est impossible. Si par contre nous privilégions la source, la métaphore est vue comme un processus unitaire, dont les issues multiples se justifient facilement.

Dans mon article, je vais d'abord souligner la pluralité des types que la métaphore connait, pour me pencher ensuite sur le transfert et sur l'interaction qu'il déclenche, et montrer finalement que, à partir de l'interaction, il est possible de justifier et d'inclure au sein d'une vision unitaire le large éventail de types documentés à travers les textes et privilégiés dans la tradition.

\section{LES ISSUES MULTIPLES DE LA MÉTAPHORE}

Si nous envisageons l'éventail des issues admises par le procès métaphorique, la nébuleuse des types attestés se partage en deux grandes familles sur la base de deux paramètres, à savoir la cohérence conceptuelle et le statut sémiotique. En premier lieu, nous pouvons différencier les métaphores cohérentes, solidement installées dans notre pensée spontanée, des métaphores conflictuelles qui la défient et la sollicitent. 
Sur le plan du statut sémiotique, les premières se justifient de l'intérieur de la pensée cohérente et coïncident avec le signifié codé et partagé de mots et d'expressions; les secondes reposent sur la capacité des formes linguistiques d'imposer un moule à la pensée, et coïncident avec des interprétations discursives contingentes d'énoncés aux signifiés conflictuels (voir plus loin la section 1.2).

\subsection{Concepts cohérents et signifiés conflictuels}

Les métaphores cohérentes se partagent entre les catachrèses (Dumarsais, [1730] 1988) et les concepts métaphoriques (Lakoff et Johnson, 1980; mais déjà Blumenberg, 1960 et Weinrich, 1958 et 1964). La catachrèse lexicale est en même temps isolée et non productive, morte. Si un bâtiment a des ailes, par exemple, cela n'implique ni qu'il ait un bec ou une queue, ni qu'il puisse voler. Au moment où il a été adapté au bâtiment, le concept d'aile a perdu toutes ses caractéristiques incompatibles avec l'identité consolidée de celui-ci. À la différence des catachrèses, les concepts métaphoriques ne sont ni isolés ni morts, mais organisés en réseaux complexes, actifs et productifs. S'il est cohérent de penser au désir comme si c'était du feu, par exemple, on peut développer cette idée avec une certaine systématicité, et dire qu'il brûle, ou qu'il est ardent, qu'il s'allume ou qu'il s'éteint, et ainsi de suite.

Comme tout concept cohérent, les concepts métaphoriques spontanés sont accessibles indépendamment de leur expression linguistique. Ils ne dépendent d'aucune en particulier, et peuvent être exprimés par plusieurs. Si l'on privilégie les types cohérents, comme il arrive dans les métaphorologies cognitives, on se fait l'idée d'une sorte de "génération spontanée» des métaphores, lesquelles pousseraient toutes seules d'un sol conceptuel déjà riche en métaphores. Le rôle des formes linguistiques apparaît comme purement instrumental, se réduisant à l'expression, à la stabilisation et à la mise en circulation de concepts indépendants (Lakoff et Johnson, 1981; Lakoff et Turner, 1989; Gibbs, 1994).

Les métaphores conflictuelles, qui forment la partie la plus qualifiée des métaphores dites vives, naissent en dehors du territoire de la cohérence.
Tout en incluant une couche importante de concepts métaphoriques, la pensée n'est pas en mesure de franchir la barrière de la cohérence sans le support actif d'un moule linguistique indépendant des contraintes de la pensée. En présence d'une métaphore vive, le rapport entre structures de la pensée et expressions linguistiques se renverse. La métaphore n'est pas simplement exprimée mais véritablement construite par l'expression.

Grâce à un réseau solide de relations grammaticales insensibles à la pression des concepts organisés, une phrase a la force de connecter les concepts atomiques dans une relation conflictuelle (Husserl, [1901] 1970); Black, 1954 et 1967; Weinrich, 1963 et 1967; Prandi, 1992, 1998 et 2004). Une expression linguistique est ainsi en mesure de construire un signifié complexe qui n'a aucune contrepartie dans le domaine des concepts cohérents, dont la légalité immanente est bouleversée. S'il est cohérent de verser des substances concrètes liquides comme le vin, et même des substances métaphoriquement liquides comme l'argent, par exemple, il n'est pas cohérent de verser la mélancolie des soleils couchants ou l'espoir, comme il arrive de le lire chez Verlaine et Baudelaire. Si un concept cohérent est simplement porté à l'expression par une forme linguistique, un concept conflictuel est réellement construit par elle. Un concept conflictuel ne peut être conçu que comme le signifié d'une expression complexe. On peut le concevoir comme une structure sémantique mais pas comme une structure conceptuelle.

Les signifiés conflictuels forment la partie la plus qualifiée des tropes, et fournissent un observatoire privilégié pour leur étude. En particulier, le conflit permet d'isoler avec précision les deux constituants d'un trope, appartenant à deux aires conceptuelles hétérogènes, que Black (1954) appelle cadre (frame) et foyer (focus). Le cadre se définit par sa cohérence avec le texte qui accueille l'expression, alors que le foyer y introduit un élément étranger. L'énoncé L'eau abreuvait [les palmiers] (Gide), par exemple, décrit une oasis: l'eau et les palmiers appartiennent au cadre cohérent, dans lequel le verbe abreuver introduit un concept étranger. 


\subsection{Signifiés et interprétations}

Cette différence profonde dans la cohérence et dans la relation avec l'expression linguistique se double d'une différence tout aussi essentielle de statut sémiotique.

Une métaphore cohérente - une catachrèse comme l'aile d'un bâtiment, mais aussi un concept métaphorique tel que l'emploi de verser avec l'argent ou de caresser avec un sentiment - est une expression pourvue d'un signifié stable qui, en principe, peut être enregistré dans un dictionnaire. Typiquement, il s'agit de l'une des acceptions d'un lexème polysémique. La métaphore est encapsulée dans ce signifié.

Le contenu d'une métaphore issue d'un conflit, tout au contraire, ne coïncide pas avec le signifié de l'expression conflictuelle, mais il est le produit contingent et réversible d'un acte d'interprétation textuelle ou discursive. Dans la métaphore vive, aucun changement de signifié ne se produit. Chaque mot, y compris le foyer, garde son signifié, et c'est précisément grâce à cela que le signifié de la phrase, et de l'énoncé qui en représente l'équivalent fonctionnel, est conflictuel. C'est le signifié conflictuel de l'énoncé pris comme un tout qui, en l'occurrence, est prêt à recevoir une interprétation métaphorique. Pour illustrer ce point, observons un exemple.

Le premier vers du célèbre poème d'Alcman - Dorment les sommets des montagnes - dépeint un procès conflictuel: il attribue l'état de sommeil, cohérent avec les êtres vivants, à la nature inanimée. Alors qu'une expression cohérente met en place un procès sans profondeur, la saturation incohérente d'un terme relationnel comme dormir donne lieu à un procès stratifié et clivé, caractérisé par un surplus de structure, c'est-à-dire de concepts et de relations, et traversé par une double tension. L'identité des montagnes est défiée par les êtres animés, qui sont les sujets cohérents du verbe dormir; en même temps, le contenu du verbe dormir est à son tour sollicité par un double virtuel cohérent avec le sujet, comme s'il était destiné à mettre en scène de façon oblique un état cohérent des montagnes. Le signifié de la phrase est ce conflit. La métaphore n'est pas encapsulée dans ce signifié; elle n'est que l'une des options multiples qui s'ouvrent à son interprétation discursive.
En premier lieu, l'énoncé admet une interprétation littérale, comme description d'un monde régi par une constitution conceptuelle différente de la nôtre. Dans les fables de Phèdre, par exemple, on rencontre des animaux et même des arbres qui parlent. La parole des arbres n'est pas métaphorique, car elle appartient de plein droit au monde de la fable:

Calumniari si quis autem voluerit,

quod arbores loquantur, non tantum ferae,

fictis iocari nos meminerit fabulis 1

Parmi les interprétations figurées, on pourrait envisager en premier lieu une option métonymique: le sommeil n'est pas attribué aux montagnes, mais aux êtres vivants qui les peuplent. L'identification d'un référent pertinent - les vivants - et sa mise en relation cohérente avec le sujet conflictuel - avec les montagnes - éteignent le conflit.

Si l'interprétation métaphorique est choisie, l'expression nous offre deux options qui peuvent se superposer, mais qui n'en demeurent pas moins logiquement indépendantes: le concept de sommeil dépeint de façon oblique un état cohérent des montagnes - par exemple, la paix ou le silence; le sommeil est appliqué aux montagnes qui, de ce fait, sont vues comme si elles étaient des êtres vivants.

À un seul signifié stable - le conflit - correspond donc une pluralité d'interprétations différentes, et la métaphore trouve sa place parmi celles-ci. Cela montre que la métaphore vive issue d'un conflit n'est ni dans le signifié d'un mot, comme la catachrèse et le concept métaphorique, ni dans le signifié d'une phrase, mais dans l'interprétation discursive de tout l'énoncé.

\section{LA SOURCE UNIFIANTE DE LA MÉTAPHORE}

2.1 Transferts de mots, transferts de concepts, interaction

Le moment unifiant de toute métaphore, par-delà les différences profondes que nous avons signalées, est dans la source. Et à la source de la métaphore se trouvent en tout cas le transfert d'un concept et l'interaction qui en découle: comme la brebis de Geoffrey de Vinsauf, qui pâture «in rure alieno", un concept se déplace dans un milieu conceptuel qui 
lui est étranger. Une fois que l'on sait qu'une brebis a sauté la haie, on sait qu'elle se trouvera dans la nécessité d'interagir avec les indigènes, mais on ne sait pas encore ce qui va lui arriver lors de cette interaction. Elle peut se rendre tout de suite, ou revenir en arrière, ou lutter pour s'imposer, avec des résultats multiples: s'intégrer paisiblement, ou imposer sa règle aux anciens maîtres de façon plus ou moins poussée. Les mêmes options s'ouvrent à un concept transféré.

Qui cherche à identifier dans le transfert le caractère spécifique et distinctif de la métaphore se heurte à une première difficulté: dans la littérature sur les tropes, le transfert est vu non pas comme le trait distinctif de la métaphore, mais comme le trait commun partagé par tous, et notamment par la métonymie et la synecdoque. Traditionnellement, ce qui est censé distinguer les trois tropes majeurs, ce n'est pas la présence ou l'absence d'un transfert, mais la nature du vecteur reliant les deux concepts engagés. L'idée traditionnelle, cependant, se base sur une équivoque qui est déjà présente chez Aristote, et notamment sur l'idée que le transfert d'un mot - l'application d'un mot à un concept différent de son contenu codé ou à un objet extérieur à son domaine de dénotation - comporte le transfert d'un concept dans une aire conceptuelle étrangère. Mais cette idée doit être remise en question.

Tant la métaphore que la métonymie et la synecdoque peuvent être décrites comme des transferts de mots ${ }^{2}$. Cependant, si nous envisageons le concept, il en va tout autrement: seule la métaphore déplace un concept dans un milieu étranger, alors que l'activation tant d'une métonymie que d'une synecdoque suppose l'identification d'une relation entre deux concepts, chacun restant solidement ancré à son milieu d'appartenance. Pour illustrer ce point, revenons à notre exemple: Dorment les sommets des montagnes.

En présence d'une interprétation métonymique, il n'y a aucun transfert du concept de sommeil du monde animé à la nature inanimée. Dans l'interprétation métonymique, le sommeil est attribué non pas aux montagnes, mais à des êtres vivants qui entretiennent une relation cohérente avec les montagnes: ils y vivent. La relation cohérente entre la nature inanimée et les êtres vivants retient le concept de sommeil dans son aire conceptuelle d'appartenance et bloque le transfert. Le sommeil reste un procès exclusif aux êtres vivants, et les montagnes restent des êtres inanimés.

En présence d'une interprétation métaphorique, le concept de sommeil, cohérent avec le monde animé, est réellement transféré dans le monde inanimé comme un élément étranger. Les montagnes se voient attribuer un procès restreint aux vivants. De ce fait, ou bien les montagnes sont vues comme si elles étaient des êtres vivants, et leur nature d'entités inanimées est remise en question, ou bien le concept de sommeil est adapté à la nature inanimée des montagnes.

Si le transfert ne caractérise que la métaphore, il est également vrai que toute métaphore, que ce soit une catachrèse, un concept métaphorique cohérent ou une métaphore vive, naît du transfert d'un concept dans un territoire conceptuel étranger. Comme le sommeil quitte le territoire des vivants pour envahir celui de la nature inanimée dans l'interprétation métaphorique du vers d'Alcman, le concept d'aile quitte le monde des animaux pour celui du bâtiment dans la catachrèse de l'aile d'un édifice, et le verbe nourrir quitte le domaine des vivants pour celui des sentiments dans son emploi avec espoir.

La présence d'un concept étranger active dans tous ces cas une double catégorisation, et donc une interaction (Black, 1954) entre concepts concurrents.

Le cas le plus transparent d'interaction métaphorique se produit chaque fois que deux concepts incompatibles, un concept qui est chez lui, pour ainsi dire, et un concept transféré, se disputent la détermination d'un seul et même objet ${ }^{3}$. Si nous utilisons l'expression larmes de pluie pour nous référer à des gouttes, par exemple, le concept de larme et le concept de goutte se disputent la détermination de ces agrégations d'eau qui tombent du ciel quand il pleut. Le concept de goutte a l'avantage d'être le gardien codé et partagé de l'identité du concept avec luimême. Le concept de larme est le concept étranger qui menace cette identité: qui peut la détruire, mais aussi la solliciter de façon créatrice. D'après Black, nous appelons sujet de discours primaire l'objet dont on parle, reconnaissable grâce à son lien stable avec un concept 
partagé - dans notre exemple, les gouttes de pluie -, et sujet de discours subsidiaire le concept étranger qui menace son identité et avec lequel il interagit ${ }^{4}$ : les larmes.

\subsection{L'interaction comme grandeur algébrique}

Quand deux concepts incompatibles se disputent la détermination d'un seul et même objet, nous pouvons imaginer deux issues opposées.

Dans un cas, le sujet subsidiaire se plie à la cohérence du sujet primaire, sans menacer son identité mais sans rien lui apporter: le solde de l'interaction est donc négatif. C'est le cas de la catachrèse. Au lieu d'enrichir le sujet primaire, le sujet subsidiaire renonce à toutes ses propriétés incompatibles avec le sujet primaire. Au lieu de faire voler un bâtiment, par exemple, ses ailes métaphoriques perdent la caractéristique d'être un instrument de vol.

Dans le cas opposé, le sujet primaire est sollicité et reformulé sous la pression du sujet subsidiaire: le solde de l'interaction est positif. En cas de solde positif de l'interaction, nous pouvons parler de projection: tout ce qu'on peut attribuer de façon cohérente au sujet subsidiaire, on a le droit de le projeter sur le sujet primaire. Un énoncé comme Le soleil versait à grands flots sa lumière sur le Mont Blanc (H.B. de Saussure), par exemple, nous autorise à voir la lumière, l'objet conflictuel de verser, comme si c'était une substance liquide, c'est-à-dire, comme si elle partageait les propriétés qualifiantes de son objet cohérent.

Au milieu, nous pouvons idéalement situer la substitution: au lieu d'appauvrir le sujet subsidiaire ou d'accepter son défi, le sujet primaire s'en défait - le solde de l'interaction est nul. Les larmes de pluie, par exemple, ne sont que des gouttes.

Si cela est vrai, l'interaction est une grandeur algébrique, compatible avec un solde négatif (la catachrèse), nul (la substitution) ou actif (la projection).

\section{StRUCTURE DE LA PROJECTION}

Ce qui distingue les différents types de métaphore - la catachrèse, les concepts métaphoriques cohérents et la métaphore vive dans toutes ses aventures, de la substitution à la projection -, c'est donc l'issue de l'interaction, son solde.

La catachrèse et la substitution sont immédiatement identifiées par le solde, respectivement passif et nul, de l'interaction, qui est dans les deux cas une valeur ponctuelle. Le solde actif, par contre, est une valeur graduée: le chemin de la projection qui s'ouvre devant une métaphore peut s'arrêter tout de suite ou se développer suivant un chemin tracé et reconnaissable, mais il peut aussi emboucher des chemins inexplorés, dont on connaît le point de départ mais pas forcément l'issue.

Face à une expression comme larmes de pluie, nous pouvons en premier lieu nous arrêter à une analogie évidente entre des larmes et des gouttes: en ce cas, le chemin de la projection s'arrête tout près de la simple substitution. Cela montre bien que le rôle de l'analogie dans l'interprétation de la métaphore, qui lui a valu sa fortune historique, consiste à arrêter le potentiel projectif dès qu'on entrevoit une propriété commune ou une ressemblance plus ou moins immédiate entre le sujet subsidiaire et le sujet primaire. Mais le potentiel créateur de la projection n'est pas limité par l'analogie.

S'il y a des larmes, nous pouvons facilement imaginer qu'il y a quelqu'un qui pleure. Or, la pluie est un procès impersonnel, sans actants: où trouver une analogie avec les pleurs? Peu importe. Insouciante de la difficulté, la métaphore nous pousse à chercher un sujet pour ces pleurs, et cela pourrait être la nature. Mais pourquoi la nature pleureraitelle? Comme c'est le jour des défunts, il est possible qu'elle éprouve de la sympathie pour nous. Donc, elle n'est pas une marraine, mais une mère qui partage notre souffrance... Le chemin de la projection nous a conduit bien loin, et l'analogie évidente entre les larmes et les gouttes nous parait bien lointaine.

Si nous voulons nous faire une idée de la latitude de la projection, les métaphores vives issues d'un conflit nous offrent le meilleur point d'observation. Quant il est interprété comme une métaphore, le conflit conceptuel n'est pas un simple détour prêt à revenir tout de suite à la cohérence, comme en présence d'une métonymie ou d'une synecdoque, 
mais se change en une source inépuisable d'énergie conceptuelle, qui semble dilater à l'infini l'horizon de la projection: on dirait à ce point qu'un silence mystique est la seule option qui nous reste. En fait, c'est le contraire. La projection métaphorique peut être circonscrite, non pas dans ses issues occasionnelles, évidemment, mais dans son périmètre conceptuel $^{5}$.

Le rôle de l'expression linguistique dans la création métaphorique se réduit au transfert d'un concept dans un domaine étranger grâce à la créativité syntaxique et au conflit que ce transfert déclenche. Une fois que le transfert a eu lieu, le témoin revient à la pensée cohérente: la projection métaphorique n'a d'autres limites que la cohérence interne du sujet subsidiaire. Tout ce qui peut être projeté dans le domaine d'accueil n'est autre chose que l'entourage cohérent du concept transféré. Circonscrire l'ensemble des projections possibles équivaut à circonscrire l'environnement cohérent et approprié du concept transféré: sa distribution. Si cette dernière tâche est raisonnable, la première l'est aussi.

L'environnement conceptuel cohérent n'a pas la même forme pour tous les types de concepts. De ce fait, le premier pas consiste à distinguer les concepts ponctuels et saturés des concepts relationnels et non saturés. Les premiers sont les contenus de noms classificatoires - livre, chien, arbre - alors que les seconds sont les contenus de verbes, d'adjectifs ou de noms de procès et qualités: rêver, chauve, naufrage ou tristesse. À première vue, on dirait que le transfert d'un nom classificatoire aboutit à une projection ponctuelle - par exemple d'un objet sur un objet -, alors que le transfert d'un concept relationnel aboutit à la projection d'une relation - par exemple, d'un procès sur un procès. En fait, les choses sont plus complexes, car ce n'est pas le concept isolé qu'on projette, mais son environnement conceptuel.

La distribution des termes relationnels se définit sur la base des arguments cohérents et appropriés qu'ils admettent, et donc en termes d'environnements formés par des concepts ponctuels. De ce fait, lors d'un transfert métaphorique, nous pouvons imaginer aussi bien la projection de la relation sur une relation étrangère que la projection de l'un de ses arguments cohérents et appropriés sur un argument étranger, ou encore un mélange des deux stratégies. Quand Gide écrit L'eau abreuvait les palmiers, par exemple, on est naturellement poussé à projeter le concept relationnel abreuver sur sa contrepartie dans le domaine végétal irriguer, mais on peut aussi se servir du transfert pour projeter sur les palmiers un troupeau de brebis, sujet solidaire d'abreuver. Quand le même auteur écrit que Des pétales neigent sur le tapis, les pétales se changent en flocons de neige ${ }^{6}$. La dimension relationnelle du contenu verbal et les propriétés ponctuelles de ses actants cohérents et appropriés s'allient pour multiplier les dimensions pertinentes de la projection métaphorique.

La distribution d'un terme ponctuel, réciproquement, se définit sur la base de l'ensemble des prédicats cohérents et appropriés qui peuvent lui être appliqués. À la différence d'un concept relationnel, un concept ponctuel ne trace aucune relation, mais il est prêt à entrer comme terme passif dans plusieurs. Cela implique que l'identité d'un concept ponctuel dépend autant du réseau de relations cohérentes et appropriées, dans lesquelles il est prêt à entrer, que de ses propriétés inhérentes (voir notamment Gross, 1992 et 1994; Le Pesant et Mathieu-Colas, 1998; Mathieu-Colas, 1998). Un livre, par exemple, n'est pas seulement un objet qui présente certaines caractéristiques observables. Il est surtout le support d'un texte que l'on peut écrire ou lire, parcourir en le feuilletant, dans lequel on trouve des renseignements, qui nous raconte une histoire ou nous introduit dans une théorie. La double identité du terme ponctuel ouvre à son tour deux chemins virtuels au transfert métaphorique, qui naturellement ne s'excluent pas mais sont prêts à se superposer. Quand l'écrivain turc N. Gürsel écrit Les cheveux de ma mère étaient un champ de blé, on pense plus immédiatement à leur couleur, et donc à une propriété inhérente, qu'à des propriétés relationnelles - par exemple qu'ils sont prêts à être moissonnés. Mais quand un monument est vu comme un livre, comme dans The Stones of Venice de Ruskin, ce sont les propriétés relationnelles du concept de livre qui sont valorisées. Si nous sommes capables de déchiffrer son écriture, de comprendre le langage dans lequel il est 
écrit et d'interpréter correctement son message, un monument devient un document (Le Goff, 1978) et, comme un texte, il nous raconte son histoire.

Si cela est vrai, l'argument projeté d'un foyer relationnel - verbe, adjectif, nom de procès ou de qualité - se change à son tour en centre d'irradiation de relations projetables, dans une réaction en chaîne qui dilate énormément les effets de la métaphore. $\mathrm{Si}$, par exemple, la lumière est versée - Le soleil versait à grands flots sa lumière sur le Mont Blanc-, elle est vue comme si elle était une substance liquide, prête à hériter de toutes les propriétés d'une substance liquide, et à entrer dans tous les procès dans lesquels une substance liquide peut entrer. Toutes ces figures de la lumière liquide sont documentées dans la poésie du Romantisme anglais et du Symbolisme français:

They pour fresh light (Shelley); The moon rains out her beams (Shelley); The light that flow'd down on the winds (Blake); The light which streams here (Byron); The free heaven [...] rains fresh light and dew / On the wide earth (Shelley); Liquid streams of light (Shelley); The floods of light / Which flow over the world (Shelley); The sunset's sea of beams, / Whose golden waves [...] / Fade fast (Shelley); Lo! The light of the morning is flowing / Through radiant portals of gold (Charlotte Brontë).

Le ciel bas et lourd [...] / il nous verse un jour noir plus triste que les nuits (Baudelaire); L'astre coule et fait un ruisseau (Leconte de Lisle); Il est étendu dans l'herbe, [...] / pâle dans son lit vert où la lumière pleut (Rimbaud); La pleine lune s'étalait, / et la solennité de la nuit, comme un fleuve, sur Paris dormant ruisselait (Baudelaire); La lune est dans son plein, / d'une blanche lueur la clairière est baignée (V. Hugo); Torrent de lumières prodiguées dans un commun et rapide écoulement (Michelet); Comme un flot ruisselant [...] de lumière (De Banville); L'astre coule et fait un ruisseau (Leconte de Lisle).

Dans tous les cas examinés, ce qui ouvre à la métaphore l'horizon de la création n'est pas la transgression de la légalité conceptuelle, qui n'est qu'un premier mouvement, mais la projection de ce même système de restrictions distributionnelles que nous aurions pu imaginer, un peu hâtivement, avoir transgressé. La créativité de la métaphore n'est pas à chercher dans ce qui est projeté, en l'occurrence un réseau de concepts cohérents et familiers, mais dans le transfert de ce réseau dans un domaine cible étranger.

\subsection{Projection et analogie}

L'exemple qui nous a permis d'introduire le concept d'interaction - l'expression larmes de pluie - avait l'avantage d'identifier immédiatement les deux termes engagés, à savoir le sujet primaire et le sujet subsidiaire. Or, parmi les métaphores vives, il arrive facilement que le sujet primaire ne soit pas accessible en dehors d'une interprétation métaphorique contingente. Si nous interprétons l'énoncé Dorment les sommets des montagnes comme l'expression métaphorique d'un état des montagnes, par exemple, on s'aperçoit tout de suite qu'on ne dispose pas d'une caractérisation univoque de cet état indépendamment d'une interprétation métaphorique donnée. Tout ce que nous savons, c'est que l'état des montagnes qu'on appelle sommeil est cohérent avec les montagnes et non pas avec les êtres vivants. Il est clair que l'identification d'un sujet primaire comporte la disponibilité d'un double virtuel, et donc d'un substitut potentiel cohérent du foyer. Toutefois, la disponibilité d'un double virtuel du foyer n'est pas garantie pour toutes les métaphores, et dépend des propriétés structurales de l'expression (Prandi, 1992: 118-122). Cette circonstance nous permet de mesurer la distance entre l'idée de projection et la notion traditionnelle d'analogie.

L'inaccessibilité du sujet primaire est un obstacle infranchissable si nous pensons que la métaphore se fonde sur une analogie, mais n'oppose aucune difficulté à la projection. Alors que l'analogie, quel que soit son contenu, est par définition une relation dont la mise en ouvre présuppose deux termes identifiables indépendamment, la projection n'a pas besoin d'une identification préalable et univoque du sujet primaire. En effet, elle peut avoir comme cible tant un sujet primaire identifié indépendamment, destiné à être restructuré, qu'une inconnue au sens mathématique, dont le contenu est destiné à être rempli par la projection elle-même. Nous n'avons aucune idée, avant la métaphore, de ce que c'est que le sommeil des montagnes. Le mécanisme de la projection est en mesure de remplir ce vide. 


\section{LIMITES DE LA PROJECTION}

Le réseau de concepts formés par la distribution cohérente d'un concept source définit le périmètre extérieur de ce qui peut être projeté, mais n'est pas en mesure de prévoir, heureusement, ce qu'il est pertinent de projeter dans un cas particulier, qui est une option contingente justifiée par des raisons contingentes. L'idée qu'une théorie de la métaphore doit prévoir une option, dont la pertinence ne peut qu'être contingente, est une idée déroutante, qui, prenant comme modèle la démarche régressive conduisant à la catachrèse, prétend lire dans le signifié de l'expression une pensée qui lui échappe, et bloquer à jamais l'énergie libérée par le conflit. Comme l'écrit Davidson: "The common error is to fasten on the contents of the thoughts a metaphor provokes and to read these contents into the metaphor itself ([1978] 1984 : 261)? .

Face à cet horizon potentiellement très ouvert, il s'agit maintenant de justifier les limites imposées à la projection, d'une part dans le contenu des concepts métaphoriques documentés dans le lexique et, d'autre part, dans l'interprétation des métaphores vives actives dans les textes. En effet, la projection métaphorique est soumise à deux ordres de contraintes. Un premier ordre de contraintes est systématique et fonde la distinction entre concepts métaphoriques cohérents et métaphores vives. Son critère est la cohérence conceptuelle du sujet primaire. Un second ordre de contraintes est contingent et adapte l'interprétation des métaphores vives au texte ou au discours qui les accueille. Son critère est la cohérence textuelle.

\subsection{La cohérence conceptuelle:}

les concepts métaphoriques partagés

Les distinctions basées sur le solde de l'interaction sont excentriques par rapport aux paramètres de la cohérence et du statut sémiotique. En particulier, les concepts métaphoriques partagés, tout en étant cohérents et faisant l'objet d'une expression passive, rentrent dans l'aire de la projection, à côté des métaphores vives.

Comme les catachrèses, les concepts métaphoriques ne sont pas l'issue contingente d'actes d'interprétation, mais des signifiés codés et stables de mots ou d'expressions, qui sont par définition cohérents. Quand on applique à l'argent des verbes comme verser ou évaporer, il n'y a aucun conflit. À la différence de la catachrèse, cependant, les concepts métaphoriques partagés présentent un solde actif de l'interaction. Quand nous disons que l'argent se verse et coule, qu'il s'évapore ou qu'il est congelé, nous ne sommes pas en présence d'innocentes façons de parler qui n'engagent pas la pensée, mais d'une aire conceptuelle compacte qui fonctionne à partir d'un modèle étranger: l'argent est réellement pensé comme si c'était une substance liquide. Donc, il y a projection.

En même temps, cette véritable inondation conceptuelle qu'est la projection n'est pas libre, comme en présence de métaphores vives, mais elle est soumise à une contrainte sévère et infranchissable: la contrainte de la cohérence du sujet primaire. Les concepts partagés sont par définition cohérents. Le codage et le partage impliquent la cohérence et bloquent toute inférence potentiellement conflictuelle issue du sujet subsidiaire. Après avoir manié de l'argent liquide, par exemple, nous n'allons pas nous essuyer les mains. Du temps, également, nous pouvons dire qu'il s'écoule. Mais cela ne nous autorise pas à parler de flots $d u$ temps, de flots des années et d'ondes $d u$ temps. Si nous le faisons, comme le fait Hölderlin, nous franchissons la limite du codage et du partage, et donc la barrière qui sépare le signifié d'une expression d'un conflit conceptuel ouvert à l'interprétation métaphorique.

Un cas privilégié de cette cooccurrence de projection et d'impératif de cohérence du sujet primaire est offert par les concepts métaphoriques d'invention dont est riche l'histoire des sciences. L'idée que la lumière se propage en ondes ne se retrouve pas seulement parmi les poètes, mais aussi parmi les physiciens: elle parcourt l'histoire de la physique depuis Huygens (XVII ${ }^{e}$ siècle), et reçoit sa formulation rigoureuse par Maxwell (fin XIXe siècle). Les métaphores de la découverte partagent avec les métaphores poétiques le défi aux idées acquises et la nature projective. Elles s'en différencient du fait qu'elles sont destinées à se changer en concepts stables et cohérents, ce qui limite le mécanisme de projection. 
Les ondes de lumière ne nous baignent pas de leurs embruns.

\subsection{La cohérence et la pertinence textuelle:}

la métaphore vive dans le texte

Dans les textes, la projection peut être sévèrement limitée par la contrainte interne et contingente de la cohérence textuelle.

Comme Conte ([1988] 1999: 29) le rappelle, la cohérence textuelle, en anglais coherence, ne doit pas être confondue avec la cohérence conceptuelle, en anglais, consistency, qui est à la base de l'idée de conflit. La cohérence conceptuelle est une propriété négative du signifié d'une phrase isolée et se réfère à l'absence de contradiction ou de conflit conceptuel dans sa structure. La cohérence textuelle, par contre, est la propriété positive de la relation entre un énoncé et le milieu communicatif qui l'accueille, typiquement un texte. La cohérence conceptuelle dépend d'un système de critères externes définis a priori: du principe de contradiction pour la composante formelle, et d'une véritable «grammaire des concepts» (Prandi, 2004 : chap. 8) pour la composante substantielle. La cohérence d'un texte dépend non pas d'un système de critères externes définissables a priori, mais de critères internes, aussi contingents que la configuration textuelle elle-même. Un texte n'est pas cohérent du fait qu'il respecte une sorte de grammaire, mais si et seulement si les énoncés qui le composent s'intègrent mutuellement, c'est-à-dire s'ils peuvent être interprétés comme coopérant à un but communicatif commun. Cela vaut aussi pour les énoncés métaphoriques.

L'adaptation d'un contenu métaphorique au texte qui l'accueille présente deux volets de signe opposé. Pour pouvoir entrer dans un texte cohérent, l'interprétation métaphorique d'un conflit conceptuel est obligée de laisser tomber toutes les projections qui ne sont pas pertinentes pour la progression thématique de ce texte. Pour les mêmes raisons, le phénomène opposé est aussi documenté: l'impératif de pertinence et cohérence qui préside à la construction d'un texte est en mesure de réveiller un concept métaphorique cohérent et même une catachrèse, déclenchant un mouvement projectif dans le second cas, lui ouvrant des chemins inattendus dans le premier.

Isolée du texte auquel elle appartient, une phrase comme Finalement l'Océan, cet ami généreux des malheureux, ouvrit ses mains pour l'accueillir admet une grande quantité d'interprétations métaphoriques. Mais quand le lecteur du roman Tom Jones de Fielding tombe sur ce passage, il ne peut pas se soustraire à la question sur le contenu ultime de la métaphore: qu'estil exactement arrivé à Tom Jones? L'interprétation offerte par Fielding lui-même - Pour m'exprimer sans figure, il décida de s'embarquer - est cohérente avec l'intrigue du roman et, de ce fait, bloque localement la dérive projective déclenchée par le conflit.

Face à une phrase comme La vérité est [...] une lumière, on est tenté de reconnaître tout simplement le concept métaphorique partagé, hérité d'une tradition millénaire. Mais quand Tocqueville écrit que La vérité est [...] une lumière que je crains d'éteindre en l'agitant, on se rend compte qu'on ne projette pas sur la vérité la propriété attendue et à son tour métaphorique de la clarté, mais la vulnérabilité, et donc une propriété de la lumière qui n'est pas incluse dans les schémas hérités. La vérité est une lumière parce qu'il est aussi facile de l'éteindre et, pour la préserver, il faut prendre des précautions. Dans les deux cas, la fragilité entraîne l'assomption de responsabilités, et ainsi de suite. De facteur de blocage, la cohérence textuelle s'est changée en facteur de promotion de la projection.

\section{CONCLUSION}

De la catachrèse à la projection la plus hardie, la métaphore est une, mais ses issues sont multiples. L'unité est dans le transfert et l'interaction; la pluralité est dans les issues multiples que le transfert et l'interaction admettent. Pour mesurer avec exactitude la nature et le contenu de chacune de ces issues, j'ai proposé de définir l'interaction, que Black (1954) oppose tant à la substitution qu'à la catachrèse, comme une grandeur algébrique qui inclut la substitution et la catachrèse comme cas particuliers aux côtés de la projection. 


\section{NOTES}

1. «Si quelqu'un voulait nous calomnier du fait que non seulement les bêtes, mais même les arbres ont le don de la parole, qu'il sache que nous nous amusons avec des fables de fantaisie" (nous traduisons). 2. L'idée d'un échange de mots se trouve dans l'étymologie même du nom grec metonymia. L'association de métonymie et transfert est courante dans la littérature, de Bonhomme (1987; 2006), qui parle de "transferts métonymiques", à Barcelona (2000: 4), qui va jusqu'à parler de projection: la métonymie est «a conceptual projection whereby one experiential domain (the target) is partially understood in terms of another experiential domain (the source) included in the same common experiential domain". (Notre traduction: "une projection conceptuelle par laquelle un domaine empirique (la cible) est partiellement compris à partir d'un autre domaine empirique (la source) inclus dans le même domaine d'expérience»).

3. Dans ce contexte, le terme objet n'est pas utilisé dans l'acception restreinte de "chose", mais dans le sens épistémologique, qui inclut tout type d'entité qui peut faire l'objet d'une catégorisation conceptuelle, et donc aussi bien des individus et des masses que des procès et des propriétés.

4. La relation entre cadre et sujet de discours primaire, d'une part, foyer et sujet de discours subsidiaire, d'autre part, est complexe et variable, et dépend de la catégorie grammaticale et de la fonction du foyer (voir Prandi, 1992: 127-134).

5. Dans ce qui suit, nous reprenons en partie le propos déjà tenu dans Prandi (2008: 36-38).

6. Cette propriété des termes relationnels métaphoriques est soulignée par Christine Brooke-Rose (1958: 241), qui s'inspire de Geoffrey de Vinsauf.

7. «La faute usuelle consiste à viser tout de suite le contenu de la pensée provoquée par la métaphore et à lire ce contenu dans la métaphore elle-même" (nous traduisons).

\section{RÉFÉRENCES BIBLIOGRAPHIQUES}

ARISTOTE [1965]: Poétique, Paris, Les Belles Lettres.

Barcelona, A. [2000]: Metaphor and Metonymy at the Crossroads. A Cognitive Perspective, Berlin et New York, Mouton de Gruyter.

Black, M. [1954]: «Metaphor ", Proceedings of the Aristotelian Society, no 55, 273-294 (réimpr. dans M. Black [1962], 25-47);

[1962]: Models and Metaphors, Ithica et Londres, Cornell University Press;

[1979]: «More about metaphor», dans A. Ortony (dir.), Metaphor and Thought, Cambridge, Cambridge University Press, 19-43.

Blumenberg, H. [1960]: Paradigmen zu einer Metaphorologie, Bonn, Bouvier \& Co.

Bonhomme, M. [1987]: Linguistique de la métonymie, Berne, Peter Lang; _- [2006]: Le Discours métonymique, Berne, Peter Lang. BROOKE-ROSE, C. [1958] : A Grammar of Metaphor, Londres, Secker \& Warburg.

CONTE, M.-E. [(1988) 1999]: Condizioni di coerenza, Florence, La Nuova Italia (nouvelle édition dirigé par B. Mortara Garavelli, Alexandrie, Edizioni dell'Orso).

DAVIDSON, D. [(1978) 1984]: "What metaphors mean", Critical Inquiry, $\mathrm{n}^{\circ} 5$ (réimpr. dans D. Davidson, Inquiries into Truth and Interpretation, Oxford, Clarendon Press, 245-264).
Dumarsais, C. [(1730) 1988]: Des tropes, ou des différents sens, Paris, Flammarion.

FONTANIER, P. [1968]: Les Figures du discours, Paris, Flammarion (rassemble le Manuel classique pour l'étude des tropes [1821] et le Traité général des figures de discours autres que les tropes [1827]).

GenetTe, G. [1966] : Figures, Paris, Seuil,

[1968]: «Introduction» à P. Fontanier, Les Figures du discours, Paris, Flammarion, 5-17.

GiBBS, R.-W. Jr [1994]: The Poetics of Mind, Cambridge, Cambridge University Press.

GROSS, G. [1992]: «Forme d'un dictionnaire électronique», dans A. Clas et H. Safar (dir.), L'Environnement traductionnel, Québec, Presses de l'Université du Québec, 255-271;

[1994]: «Classes d'objets et description des verbes", Langages, $\mathrm{n}^{\circ} 115,15-30$.

GROUPE $\mu$ [1970]: Rhétorique générale, Paris, Larousse.

HUSSERL, E. [(1900) 1961]: Logische Untersuchungen, vol. I (1900) et vol. II (1901), Halle, Niemeyer. Édition critique: Husserliana, vol.XVIII (1975) et vol.XIX, I-II (1984), La Haye, Nijoff. Trad. française (de la 2e éd., Halle, 1922-1923): Recherches logiques, tome I (1959) et tome II (1961-1962), Paris, PUF.

LAKOFF, G. et M. JOHNSON [1981]: Metaphors we Live by, Chicago et Londres, The University of Chicago Press.

LAKOFF, G. et M. TURNeR [1989]: More than Cool Reason, Chicago et Londres, The University of Chicago Press.

LE GOFF, J. [1978]: «Documento/Monumento », Enciclopedia Einaudi, vol. V, Turin, Einaudi.

Le Pesant, D. et M. Mathieu-Colas (dir.) [1998]: Les Classes d'objets, Langages, ${ }^{\circ} 131$, Paris, Larousse.

MATHIEU-COlaS, M. [1998]: « Illustration d'une classe d'objets: les voies de communication", dans D. Le Pesant et M. Mathieu-Colas (dir.), 77-90.

PRANDI, M. [1992]: Grammaire philosophique des tropes, Paris, Minuit; [1998]: "Contraintes conceptuelles sur la distribution: réflexions sur la notion de classe d'objet", dans D. Le Pesant et M. Mathieu-Colas (dir.), 34-44;

[2004]: The Building Blocks of Meaning, Amsterdam et

Philadelphie, John Benjamins;

[2008]: « La métaphore: la pensée cohérente à l'épreuve du conflit conceptuel», dans D. Jamet (dir.), Dérives de la métaphore, Paris, L'Harmattan, 31-34.

RichaRDS, I. A. [1936]: The Philosophy of Rhetoric, Oxford, Oxford University Press.

TOdorov, T. [(1970) 1979]: «Synecdoques», Communications, nº 6 (réimp. dans T. Todorov, W. Empson, J. Cohen, G. Hartman, F. Rigolot (dir.), Sémantique de la poésie, Paris, Seuil, 7-26).

VInSAUF, G. de [1924]: «Poetria Nova», dans E. Faral, Les Arts poétiques $d u$ XII et $d u$ XIII siècles, Paris, Champion.

WeINRICH, H. [1958] : «Münze und Wort. Untersuchungen an einem Bildfeld", Romanica, Festschrift Rohlfs, Halle, Niemeyer, 508-521; [1963]: «Semantik der kühnen Metapher », Deutsche Vierteljahrsschrift für Literaturwissenschaft and Geistesgeschichte, n $37,325-344$; [1964]: «Typen der Gedächtnismetaphorik», Archiv für Begriffsgeschichte, $\mathrm{n}^{\circ}$ IX, 23-26; [1967]: «Semantik der Metapher », Folia Linguistica, n 1, 3-17. 